\title{
Standardization of Isolated Caudate Lobectomy by Extrahepatic Glissonean Pedicle Isolation and HV Root - At First One-way Resection Based on Laennec's Capsule: Open and Laparoscopic Approaches
}

\author{
Yutaro Kato, Atsushi Sugioka, Yoshinao Tanahashi, Masayuki Kojima, Sanae Nakajima, Akira Yasuda, \\ Jun-ichi Yoshikawa, Ichiro Uyama
}

Department of Surgery, Fujita Health University, Toyoake, Aichi, Japan

\section{ABSTRACT}

Isolated caudate lobectomy (ICL) is technically demanding and its surgical techniques are not standardized. Herein, we describe our method of open and laparoscopic ICL by the extrahepatic Glissonean pedicle approach (GPA) and hepatic vein (HV) root-at first one-way parenchymal resection, which are both based on Laennec's capsule. Firstly, all the Glissonean pedicles of the caudate lobe are isolated and divided extrahepatically without parenchymal dissection. We have devised two different techniques for the extrahepatic pedicle control. One is 'the central hilar technique', where all the major hilar pedicles are utilized to isolate caudate pedicles, and the other is 'the left-to-right tracking technique', where the caudate pedicles are serially divided along the hilar plate from the left to the right sides. After controlling all pedicles of the caudate lobe, it is mobilized from the vena cava. Parenchymal dissection starts from exposing the roots of left, middle and right hepatic veins and the dorsal surface of their walls are tracked by dissection in the cranio-caudal direction. Parenchymal resection is completed at the hilar plate and right-side demarcation line. In conclusion, ICL, either open or laparoscopic, can be standardized by the extrahepatic GPA and HV-root at first one-way parenchymal resection based on Laennec's capsule.

Key words: isolated caudate lobectomy, laparoscopic liver resection, Glissonean pedicle, Laennec's capsule, plate system, hepatic vein

\section{INTRODUCTION}

Resection of liver tumors in the caudate lobe is technically demanding because of its deep location, proximity to major hepatic veins (HVs) and inferior vena cava (IVC) and the anatomical variety of the feeding vessels. Therefore, for caudate tumors that require a total caudate lobectomy, it tends to be combined with other hepatectomy procedures to seek technical feasibility and sufficient surgical margins $(1,2)$. However, in some patients with impaired hepatic functional reserve, these procedures are not tolerable and only isolated caudate

\author{
Corresponding author: \\ Yutaro Kato, MD \\ Department of Surgery \\ Fujita Health University \\ 1-98, Dengakugakubo \\ Kutsukake-cho, Toyoake \\ Aichi, 470-1192, Japan \\ Tel: +85-562-93-9254, \\ Fax: +85-562-93-7060 \\ E-mail: y-kato@fujita-hu.ac.jp
}

\author{
Abbreviations: \\ ICL: isolated caudate lobectomy \\ GPA: Glissonean pedicle approach \\ $\mathrm{HV}$ : hepatic vein \\ IVC: the inferior vena cava \\ LHV: the left hepatic vein \\ MHV: the middle hepatic vein \\ RHV: the right hepatic vein \\ G-R: the right Glissonean pedicle \\ G-L: the left Glissonean pedicle \\ G-ant: the Glissonean pedicle \\ of the anterior section of the liver \\ G-post: the Glissonean pedicle \\ of the posterior section of the liver \\ G1c: the Glissonean pedicle of the \\ caudate process of the caudate lobe \\ G1L: the Glissonean pedicle of the \\ Spiegel lobe of the caudate lobe \\ G1r-L: the Glissonean pedicle \\ of the left part of the paracaval portion \\ of the caudate lobe \\ G1r-R: the Glissonean pedicle of the \\ right part of the paracaval portion of \\ the caudate lobe \\ ArP: the Arantius plate
}

Received: 18.02 .2020

Accepted: 07.04.2020

Copyright ( $\odot$ Celsius Publishing House www.sgo-iasgo.com 
lobectomy (ICL) is indicated from both a parenchymalsparing perspective and a curability point of view. In general, ICL is considered to be technically more difficult and less standardized than a caudate lobectomy in combination with the other adjoining part of the liver.

According to corrosion liver cast studies by Kumon, the caudate lobe is composed of three parts: Spiegel lobe, paracaval portion and caudate process portion (3), all of which need to be resected en bloc during anatomic ICL. Although there have been many descriptions of non-anatomic partial resections of the caudate lobe, reports on surgical techniques of completely anatomic ICL are limited. One of them is the 'high dorsal resection' method, which is based on the determination of the boundaries of the caudate lobe using ultrasound-guided dye staining and on the exposure of middle (MHV) and right (RHV) hepatic veins from their roots (4). Another is the transhepatic approach, where the caudate lobe is exposed by transecting the liver parenchyma along the main portal fissure and opening the hepatic hilum anteriorly (5). Although several authors have reported the laparoscopic approach to ICL with varied techniques $(6,7)$, the technical feasibility and safety of the laparoscopic ICL are not established.

In this article with a video presentation, we describe a novel method of anatomic ICL based on the combination of the inflow control by the extrahepatic Glissonean pedicle approach (GPA) and the outflow control by an approach with the HV-root at first craniocaudal, one-way parenchymal resection. Both of the two techniques are based on the anatomical background of Laennec's capsule (8).

\section{SURGICAL TECHNIQUES}

For the open ICL, a reversed L-shape incision is made on the upper-middle abdomen, and the patient position and trocar placement for the laparoscopic approach are described later in the 'Preparation for laparoscopic ICL' section. In our method of ICL, we have devised two different techniques for extrahepatic GPA to isolate the Glissonean pedicles of the caudate lobe for ICL. One is 'the central hilar technique' and the other is 'the left-to-right tracking technique'. The procedures in the former technique are as follows. The first step is 'the cystic plate cholecystectomy', in which the cystic plate is detached from the Laennec's capsule of the liver parenchyma of the gall bladder bed during cholecystectomy (8). The cystic plate cholecystectomy is useful as it allows a direct access to the surface of the Glissonean pedicle of the anterior section. The second step is isolation of the hepatoduodenal ligament, right (G-R) and left (G-L) pedicles, anterior (G-ant) and posterior (G-post) pedicles, caudate process pedicle (G1c) and Aranthius plate (ArP), according to Laennec's capsule-based layer dissection and Gate theory-guided access to the hilum (8). The left lateral section is mobilized and flipped up toward the right side over the segment IV. Caudal approach helps you sling a tape around the Spiegel lobe pedicle (G1L), for which lifting up of both tapes around G-L and ArP is useful. Then, the left stump of the tape holding $\mathrm{G}-\mathrm{L}$ is passed under $\mathrm{G} 1 \mathrm{~L}$ and then ArP one by one, which ends up with isolating the pedicles to the left part of the paracaval portion (G1r-L) extrahepatically. This method of isolating deep pedicles by switching tapes is what we call 'the subtraction method'. In the same way, the pedicles to the right part of the paracaval portion (G1r-R) can be isolated using the subtraction method by switching the tape passed around G-R over G1c, G-post and finally G-ant. In some cases, $\mathrm{G} 1 \mathrm{r}-\mathrm{R}$ derives from $\mathrm{G}-\mathrm{L}$, where $\mathrm{G} 1 \mathrm{r}-\mathrm{R}$ can be isolated by switching the tape of G-L over G1L, ArP and G1r-L one by one, using the subtraction method. These serial procedures enable complete extrahepatic securement of all the Glissonean pedicles of the caudate lobe. By dividing or clamping all the pedicles, the isolated caudate lobe is entirely devascularized before liver parenchymal resection.

The other technique for extrahepatic pedicle isolation and division for $\mathrm{ICL}$ is what we call the left-to-right tracking technique'. The procedures using this technique start from the extrahepatic isolation of G1L and ArP. After division of G1L and ArP on the hilar side, you can make an access to the cranio-dorsal face of the hilar plate from the left side. Without parenchymal dissection, you can stick to the layer along the hilar plate to identify all the caudate pedicles coming off the plate. Isolation and caudal traction of G-L and G-R are useful for making a space between the hilar plate and the confronting liver parenchyma. During such hilar dissection in the left-toright direction, you can identify G1r-L first and then G1c and G1r-R. Those pedicles are isolated and divided one by one from the left side until you reach the cranial surface of the bifurcation of G-ant and G-post. These procedures enable you to divide all the caudate pedicles extrahepatically. All of these procedures in both of the two different techniques can be appropriately performed in the laparoscopic setting as well.

Following the complete pedicle control, the caudate lobe is fully mobilized from IVC. In the open ICL, the liver is completely mobilized from both right and left sides, whereas in the laparoscopic ICL, all the mobilization procedures can be carried out from the left lateral 
side owing to the good caudal view in an ample surgical field. The ventral border of the paravcaval portion is considered to be the dorsal surface of the walls of the left hepatic vein (LHV), MHV and RHV. Following division of ArP at its attachment to LHV or IVC, parenchymal resection starts from the roots of the LHV and MHV to the peripheral side in the cranio-caudal and left-to-right direction, which is what we call ' $\mathrm{HV}$ root-at first one-way resection'. In the laparoscopic approach, during exposure of the dorsal surface of the LHV and MHV walls, the magnified view is helpful for safe dissection. Several hepatic venous tributaries from the caudate lobe joining the LHV and MHV are divided. Dissection of the roots of LHV and MHV and the anterior surface of IVC at the HV confluence, allows exposure of the root of RHV, which is tracked to the peripheral side by cranio-caudal parenchymal resection. We normally use an ultrasonic surgical aspirator for hepatic parenchymal transection, in either open or laparoscopic approach. The demarcated borders between the paracaval portion and the right liver and that between the caudate process portion and the right posterior section are dissected. Finally, ICL is completed on the hilar plate. We normally do not use Pringle maneuver during ICL. The specimen is removed from below G-R or from the left-side opened space. The post-resection view shows the empty area surrounded by the exposed three major hepatic veins, IVC and the denuded hilar plate with resected stumps of the Glissonean pedicles of the caudate lobe.

\section{Preparation for laparoscopic ICL}

In laparoscopic ICL, the patient is set at the supine position with the legs apart. The surgeon basically stands on the left side of the patient and changes the position to the right side during dissection and division of G1c and parenchymal dissection between the caudate process portion and the posterior section. The endoscope port is placed at the umbilicus, and five 12-mm working trocars are placed at the epigastrium, the left subcostal point on the mid-clavicular line and the right subcostal point on the pre-axillary line and the right and left points on the mid-clavicular line in the mid abdomen. During hilar dissection, it is important that the liver-side stump of the divided round ligament is lifted up to the epigastrium for ensuring a wide surgical field at the hilum. During parenchymal dissection, the left lateral section is kept at the rightward overturned position.

\section{DISCUSSION}

Surgical techniques of anatomic ICL are not standardized, not only because of a difficulty in determining the borders between the caudate lobe and the adjoining segments but also because of the surgical difficulties due to its deep location and proximity to the major HVs and IVC. In accordance with the Kumon's anatomy of the caudate lobe (3), anatomic ICL means an en bloc resection of its composing 3 portions: Spiegel lobe, paracaval portion and process portion. There are controversies in regard to the anatomical borders between the paracaval portion and the adjoining liver segments or between the caudate process portion and the posterior section of the liver. Takayama et al. suggested that these borders should be MHV and RHV (4), which also serve as approachable landmarks for parenchymal resection. On the other hand, the Kumon's caudate anatomy is based on the portal venous and biliary branch ramifications. In our opinion, the anatomical caudate lobe is the liver area supplied by the portal pedicles deriving from the hilar plate, which may be included in the concept of Kumon. The accurate borders between the paracaval portion and other segments remain ambiguous, depending on the anatomical concepts, but assigning the upper part of $\mathrm{MHV}$ and $\mathrm{RHV}$ to the landmarks for parenchymal resection may be surgically practical and might increase technical safety.

Based on our above-mentioned concept of anatomical caudate lobe, we devised a new technique of ICL that is composed of the Laennec's capsulebased extrahepatic GPA and HV-root at first one-way parenchymal resection. We further devised two different techniques of GPA for caudate pedicle isolation. The most characteristic procedure in the 'the central hilar technique' is that as the direct extrahepatic isolation of the paracaval portion pedicles is highly difficult, we indirectly isolate them by repeating the subtraction method. The paracaval portion pedicles should eventually be those remaining after subtracting G1c, G-post and G-ant from G-R on the right side, or after subtracting G1L and ArP from G-L on the left side. Such serial isolation of pedicles using the subtraction method should be difficult without utilizing Laennec's capsule-based pedicle dissection. On the other hand, in 'the left-to-right tracking technique', all the caudate pedicles are isolated and divided from the left side before starting parenchymal dissection. This technique may be simpler and easier than the former technique because this does not need serial hilar pedicle isolation using the subtraction 
method (or even cholecystectomy). However, in this technique, the limitation of the space between the hilar plate and the paracaval portion pedicles is a major concern. Therefore, from our experience, this technique is much more suitable for the laparoscopic approach than for the open setting, because the tight space at the dorsal side of G-L and the hilar plate is not readily approachable without parenchymal dissection in the open procedure, whereas the laparoscopic caudo-dorsal approach provides a better view of that space from the left side without parenchymal resection.

Our approaches to inflow occlusion in ICL are considered to confer several merits. First, the complete occlusion of the inflow to the caudate lobe determines the anatomical border of the caudate lobe before parenchymal dissection and may also reduce bleeding during it, even without Pringle maneuver. Particularly in patients with severely impaired liver function, occlusion of the inflow to the to-be-resected area only could replace the long-time repeat intermittent Pringle maneuver. Second, preceding division of almost all caudate pedicles may increase the mobility of the caudate lobe during its mobilization from IVC and parenchymal dissection, thereby increasing the technical feasibility.

Anatomic ICL requires exposure of the dorsal surface of the upper part of MHV and RHV, and in previously reported techniques, they are approached during parenchymal dissection in the caudo-cranial direction from the right side $(4,6)$. On the contrary, our approach to these major HVs starts from exposing the roots of LHV and MHV followed by parenchymal dissection and tracking the dorsal surface of the HVs in the cranio-caudal, left-to-right direction, during which the root of RHV is exposed. Particularly in the laparoscopic approach, the magnified view from the left lateral side is quite useful for this procedure. We think that there are several merits in our approach of HV-root at first one-way resection. First, the roots and dorsal surface of the LHV, MHV and RHV can be securely and safely confirmed and exposed, thereby reducing the potential anatomical misjudgment. Second, as the resection plane is opened from the left cranial side, when an unexpected bleed occurs on the major HVs, it can be safely controlled in a good surgical field.

Our method of ICL is different from "High dorsal resection" technique, an established method of ICL, proposed by Takayama et al. $(1,4)$ in several points. First, our method of inflow control is the extrahepatic Glissonean approach, in which only the caudate pedicles can be occluded even before starting parenchymal resection, while they divide caudate pedicles during liver transection under repeated Pringle maneuver. Second, we confirm the borders between the caudate and other segments by ischemic demarcation after occlusion of all the caudate pedicles, while they determine the borders by the dye-staining method. Third, the direction of liver parenchymal transection in our approach is cranio-caudal from the left side, while theirs is caudo-cranial from the right side.

Our techniques for ICL may have some limitations. In cases of tumors abutting on the hilar plate with suspected invasion, the Glissonean approach should not be applied. In addition, in cases with previous hepatectomies where the hilar plate was dissected extensively, our approach may be hardly applicable and even if applicable, extremely technically demanding.

\section{CONCLUSION}

This report described a new surgical technique to perform anatomic ICL, either open or laparoscopic, and to standardize the procedure by the extrahepatic GPA and HV-root at first one-way parenchymal resection. Future studies are necessary to evaluate the safety and feasibility of this new technique. Furthermore, anatomic ICL remains a complex procedure and a learning curve is required.

\section{Disclosures}

The authors have no conflicts of interest to disclose relevant to the content of this article.

\section{REFERENCES}

1. Takayama T, Midorikawa $\mathrm{Y}$, Higaki T, Nakayama H, Moriguchi M, Aramaki 0, et al. Algorythm for resecting hepatocellular carcinoma in the caudate lobe. Ann Surg. 2019

2. Higaki T, Takayama T, Modorikawa Y. Ventral approach for resecting hepatocellular carcinoma in the caval portion of the caudate lobe. Surgery. 2018; 163(3):1245-1249.

3. Kumon M. Anatomy of the caudate lobe with special reference to portal vein and bile duct (in Japanese, with English abstract). Acta Hepatol Jap. 1985; 26:1193-1199.

4. Takayama T, Tanaka T, Higaki T, Katou K, Teshima Y, Makuuchi M. High dorsal resection of the liver. J Am Coll Surg. 1994; 179:72-75.

5. Yamamoto J, Takayama T, Kosuge T, Yoshida J, Shimada K, Yamasaki S, et al. An isolated caudate lobectomy by the transhepatic approach for hepatocellular carcinoma in cirrhotic liver. Surgery. 1992; 111(6):699-702.

6. Oh D, Kwon CHD, Na BG, Lee KW, Cho WT, Lee SH, et al. Surgical techniques for totally laparoscopic caudate lobectomy. J Laparoendosc Adv Surg Tech. 2016; 26(9):689-692

7. Salloum C, Lahat E, Lim C, Doussot A, Osseis M, Compagnon P, et al. Laparoscopic isolated resection of caudate lobe (Segment 1): $A$ safe and versatile technique. J Am Coll Surg. 2016; 222 (5):e61-e66.

8. Sugioka A, Kato Y, Tanahashi Y. Systematic extrahepatic Glissonean pedicle isolation for anatomical liver resection based on Laennec's capsule: proposal of a novel comprehensive surgical anatomy of the liver. J Hepatobiliary Pancreat Sci. 2017; 24(1):17-23. 